\title{
Ocular Adnexal Infections
}

\author{
Bryan R. Costin $\cdot$ Julian D. Perry
}

Published online: 22 July 2014

(C) Springer Science + Business Media New York 2014

Keywords Ocular adnexal infections - Blepharitis . Conjunctivitis · Dacryoadenitis · Canaliculitis ·

Dacryocystitis · Preseptal cellulitis · Orbital cellulitis

The lacrimal gland and the nasolacrimal excretory system (canaliculus, nasolacrimal sac, and nasolacrimal duct), the extraocular muscles, the eyelids, eyelashes, eyebrows, and the conjunctiva comprise the ocular adnexal structures. Infections affecting these tissues represent a major cause of annual ophthalmology visits, a significant cause of morbidity, and healthcare cost. The purpose of this report is to critically review and evaluate the literature published over the past year pertaining to ocular adnexal infections. However, an update on microbial resistance precedes any discussion of infectious diseases.

Sotonzono et al. reported cases of methicillin-sensitive and methicillin-resistant Staphylococcus aureus (MSSA, MRSA, respectively) refractory to fluoroquinolone therapy may be treated with $1 \%$ vancomycin ointment [1]. The staphylococcal ocular adnexal infections included conjunctivitis, blepharitis, meibomitis, dacryocystitis, or keratitis. However, the study was small (25 patients) and adverse reactions to the vancomycin ointment occurred in $28 \%$ of patients (7/28) and included eyelid edema and conjunctival hyperemia. Nonetheless, the importance of new antibiotics and formulations becomes clear, when one considers the emergence of antibiotic-resistant microorganisms and our obligation to prescribe antibiotic therapy responsibly.

B. R. Costin · J. D. Perry $(\varangle)$

Cole Eye Institute, Cleveland Clinic, 9500 Euclid Avenue,

Mail Code i-20, Cleveland, OH 44195, USA

e-mail: perryj1@ccf.org
Another study examined culture and sensitivity results obtained from ocular and ocular adnexal infections over a 4-year period in order to trend the emergence of levofloxacin resistance [2]. These infections ranged from orbital cellulitis, blepharitis, dacryoadenitis, conjunctivitis, infectious corneal ulcer, and endophthalmitis, and of the 242 cases reviewed, levofloxacin-resistant strains occurred in $32.8 \%$ of isolates. These data raise awareness of resistance to this fluoroquinolone and a possible future increase in levofloxacin-resistant ocular and ocular adnexal infections. Although no cases of vancomycin-resistant strains were reported, the authors advocate using this antibiotic only, when absolutely necessary to prevent the emergence of vancomycin resistance.

Several antibiotic trials for the treatment of conjunctivitis were published over the past year. Malamos et al. showed single-dose oral azithromycin to be an effective alternative to combined oral azithromycin/doxycycline therapy in the treatment of adult inclusion conjunctivitis [3]. Topical azithromycin $1.5 \%$ ophthalmic solution was shown to provide a more rapid resolution than tobramycin $0.3 \%$ ophthalmic solution in the treatment of purulent bacterial conjunctivitis in children, with a more convenient twice-a-day dosing regimen [4]. A randomized control trial comparing polymyxin B-trimethoprim to moxifloxacin showed equal response rates in purulent conjunctivitis in pediatric patients and offered significant cost saving compared to moxifloxacin [5]. Finally, besifloxacin $0.6 \%$ ophthalmic solution three times daily for 7 days was shown to be safe in patients aged 1 year and older for bacterial conjunctivitis $[6,7]$.

Conjunctivitis is arguably the most common ocular adnexal infection, and it is often difficult to determine the etiology due to the vast number of causes of this condition. Bennett et al. reported a multiplex real-time PCR assay for 
rapid and simultaneous detection of HSV 1 and 2, VZV, adenovirus, and Chlamydia trachomatis (C. trachomatis) from eye swabs was developed and evaluated [8]. They showed the assay was sensitive, specific, and robust. Moore et al. echoed the need for cost-effective molecular screening in viral conjunctivitis using a boil extraction amplification technique [9]. We agree that cultures ensure diagnosis and improve patient management and infection control measures. However, cultures can show no growth, despite the presence of pathogen and contaminants and normal flora can lead to diagnostic confusion. Nonetheless, microbiologic confirmation should be attempted when possible.

Case reports comprised the remainder of publications on the subject of conjunctivitis over the past year. Chaurasia et al. described at six cases of chronic, tuberculous conjunctivitis and highlighted the variable presentation of this rare condition and to consider this diagnosis in cases of refractory, chronic conjunctivitis. Two patients had ulcerative disease, one had a nodulo-ulcerative lesion, one had bilateral nodular epibulbar masses, and one had a hypertrophic papillary lesion. Systemic involvement may or may not be present and symptoms resolved with four-drug antituberculosis therapy [10]. Boto-de-Los-Bueis et al. reported a case of human herpesvirus 6 conjunctivitis [11].

Demodicosis, or infection with the human Demodex mites, Demodex folliculorum and Demodex brevis, predominated the infectious blepharitis literature in 2013 [13-21]. Ocular adnexal manifestations of these microscopic arachnids range from asymptomatic colonization to chronic blepharitis, chalazia, madarosis, or less commonly keratoconjunctivitis and can be confused with other inflammatory dermatoses, such as folliculitis, rosacea, and dermatitis and may even mimic malignancy [12-14]. Demodex could also be considered in the differential diagnosis of herpetic blepharitis as Yun et al. reported a case of periocular Demodex folliculitis presenting as a vesiculopustular rash [15].

More astounding than the variety of Demodex presentations is its prevalence. Hom et al. showed that $D$. folliculorum and $D$. brevis infestations and infections are underdiagnosed, undertreated, and underappreciated [16 • $]$. In their study, colonies of one to two thousand mites in $100 \%$ of patients aged 70 years and older. Patel et al. echoed the high prevalence and under-recognition of Demodex ocular adnexal infections and also highlighted the morbidity these patients frequently experience with courses of unsuccessful medications and treatments and a lack of diagnosis adding to their frustrating symptoms [17]. Similarly, Anane et al. reported seven cases of Phthirus pubis (the pubic louse) eyelash ectoparasitosis or phthiriasis palpebrarum as misdiagnosed as blepharitis [18].

Demodicosis can be diagnosed by slit-lamp examination looking for mites or the cylindrical cuffing characteristic of the mite infestation $[16 \bullet \cdot]$. Confirmation of infection can be made using light microscope evaluation of epilated lashes or rotation of the cilia within the follicle exposing the mite(s) [16••, 19]. Kosik-Bogacka found that demodicosis occurred at equal rates in the immunocompetent and the immunocompromised [13]. Treatment options range from polyhexamethylene biguanide (PHMB) eyelid scrubs, oral ivermectin and oral ivermectin-metronidazole, and tea tree oil eyelid scrubs $[15,20,21]$.

The preseptal/orbital cellulitis literature contained several interesting case reports including orbital myositis with apex syndrome due to herpes zoster ophthalmicus, preseptal cellulitis due to embedded insects, their body parts, or secretions (Megacopta centrosignatum), orbital cellulitis due to an odontogenic abscess without associated sinus disease, and Escherichia coli endogenous panophthalmitis causing a secondary orbital cellulitis [22]. Elmann et al. reported twenty-six cases of periocular abscesses occurring after eyebrow epilation, and over half of these cases were culture positive for MRSA [23]. A case of aggressive Arcanobacterium hemolyticum orbital cellulitis secondary to frontal sinusitis raised awareness of this potentially lifethreatening microorganism.

Multi-drug-resistant Pseudomonas aeruginosa and Proteus mirabilis in a 2-year-old child and a case of MRSA in a previously healthy neonate reminds us that antibiotic resistance occurs and failure of an infection to respond to therapy should warrant consideration of change in therapy or even an alternate diagnosis [24-29]. For example, the literature reminds us that refractory cases may in fact be cause to consider alternate etiologies such as malignancy and inflammatory conditions. Examples of preseptal and orbital cellulitis mimicry reported over the past year include anaplastic large-cell lymphoma, CD56-negative extranodal NK/T cell lymphoma, choroidal melanoma, acute myelogenous leukemia, and mature teratoma [30-34]. We have found that having a low threshold for reconsidering diagnosis and a multi-disciplinary approach are helpful in refractory or aggressive cases to uncover possible masquerading conditions.

Other interesting papers on the topic of preseptal and orbital cellulitis include a reminder by Upendran and McLoone to monitor children of amblyogenic age for this condition even after resolution of edema [35]. Pushker et al. performed a prospective, single-masked, interventional trial comparing patients with orbital cellulitis treated with IV antibiotics versus IV antibiotics and oral steroids [36]. They found shorter duration of symptoms and hospital stay and concluded steroid treatment poses low risk of exacerbating infection [36]. Mathew et al. retrospectively reviewed CT scans of preseptal and orbital cellulitis at a tertiary care facility over a 10 -year period to look at incidence and complications [37]. Based on their findings, they 
recommend that all patients with a suspected diagnosis of orbital cellulitis undergo CT scan of the head and the orbits with contrast with attention being paid to the paranasal sinuses, the superior ophthalmic vein, the cavernous sinus, and the intracranial structures.

Moving onto the lacrimal system and beginning with the lacrimal gland, the dacryoadenitis literature over the past year consisted mainly of case reports. Chandravanshi and Mishra reported the first case of Klebsiella pneumonia dacryoadenitis in an 11-year-old boy with a sputum culture-proven Klebsiella upper respiratory infection [38]. Toledano et al. reported a case of tuberculous dacryoadenitis as the presenting sign of human immunodeficiency virus (HIV) [39•].

The canaliculitis literature consisted of a case report of Actinomyces para-canalicular abscess mimicking a chalazion [40]. Indeed, we too have encountered medial canthal chalazia mimicking canaliculitis as well as canaliculitis mimicking peripunctal chalazia. We find that erythema and other signs of inflammation point toward chalazion, while true canaliculitis commonly lacks an overt inflammatory appearance. Nonetheless, the differential diagnosis of medial canthal lesions should include canaliculitis, chalazion, and multiple types of neoplasias.

Several interesting dacryocystitis cases were also reported over the past year including Cardiobacterium hominis, Paracoccidioides brasiliensis, Francisella tularensis, and Morganella morganii [41, 42•, 43, 44]. However, the dacryocystitis literature also contained several interesting papers describing epidemiology, outcomes, and current treatment options for this ocular adnexal infection. Ali et al. reviewed 320 cases of acute dacryocystitis presenting to a tertiary care center (ophthalmic plastic and reconstructive surgery clinic) over a 22-year period [45]. These data showed acute dacryocystitis comprises $2.4 \%$ of all patients presenting with lacrimal system abnormalities with a mean age of presentation of 37 years, female to male ratio of $2: 1$, and no difference with respect to laterality. This study supported the success rate of external dacryocystorhinostomy (DCR) in the treatment of dacryocystitis. Sun et al. studied the success rate of endoscopic DCR in the treatment of recurrent dacryocystitis and found it similar to the reported success rate of external DCR [46].

In their retrospective review, Ali et al. post-infection fistula (5.6\% of cases) formation was more commonly seen with spontaneous rupture of a lacrimal abscess $(83 \%$ of fistulas) [45]. Lacrimal sac fistulae can be a challenging condition to correct. Eshraghi et al. showed that, with early incision and draining with external DCR, lacrimal sac fistulae did not develop over a 3-year period in patients presenting with acute dacryocystitis and an abscess [47]. Another study evaluated outcomes in the surgical management of fistulous dacryocystitis cases and further supported fistula excision with DCR and bicanalicular silicon tube intubation as successful for cases of NLDO in which a fistula is already present [48].

In conclusion, the ocular adnexal are susceptible to a wide variety of infections. Review of the literature published in 2013 raises our awareness of very common contagions like demodex mites as well as a multitude of rare pathogens - some being reported for the first time. We reported a case of Candida albicans eyelid abscess in a 1-year-old child, which was eventually found to represent a lacrimal sac diverticulum with fistula, but the unusual nature of the fungal infection led to systemic work-up and revealed severe neutropenia likely consistent with cyclic neutropenia [49•]. Indeed, ocular adnexal infections provide an opportunity to diagnose potentially life-threatening immune deficiencies. [39•, 42•, 49•, 50•]. Finally, cases of refractory and/or aggressive ocular adnexal infections should prompt the clinician to reconsider the diagnosis, obtain cultures or additional, and consider involvement of other disciplines.

Disclosure Bryan R. Costin and Julian D. Perry have no conflicts of interest to report.

Human and Animal Rights and Informed Consent This article does not contain any studies with human or animal subjects performed by any of the authors.

\section{References}

Papers of particular interest, published recently, have been highlighted as:

- Of importance

- Of major importance

1. Sotozono C, Fukuda M, Ohishi M, et al. Vancomycin Ophthalmic Ointment $1 \%$ for methicillin-resistant Staphylococcus aureus or methicillin-resistant Staphylococcus epidermidis infections: a case series. BMJ Open. 2013;3:e001206. doi:10.1136/bmjopen2012-001206.

2. Shimizu Y, Toshida H, Honda R, et al. Prevalence of drug resistance and culture-positive rate among microorganisms isolated from patients with ocular infections over a 4-year period. Clin Ophthalmol. 2013;7:695-702.

3. Malamos P, Georgalas I, Rallis K, et al. Evaluation of single-dose azithromycin versus standard azithromycin/doxycycline treatment and clinical assessment of regression course in patients with adult inclusion conjunctivitis. Curr Eye Res. 2013;38:1198-206.

4. Bremond-Gignac D, Nezzar H, Bianchi PE, et al. Efficacy and safety of azithromycin $1.5 \%$ eye drops in paediatric population with purulent bacterial conjunctivitis. $\mathrm{Br} \mathrm{J}$ Ophthalmol. 2014;98:739-45.

5. Williams L, Malhotra Y, Murante B, et al. A single-blinded randomized clinical trial comparing polymyxin B-trimethoprim and moxifloxacin for treatment of acute conjunctivitis in children. J Pediatr. 2013;162:857-61. 
6. Mahvan TD, Hornecker JR, Buckley WA, et al. The Role of Besifloxacin in the Treatment of Bacterial Conjunctivitis. Ann Pharmacother. 2014;48:616-25.

7. Malhotra R, Ackerman S, Gearinger LS, et al. The safety of besifloxacin ophthalmic suspension $0.6 \%$ used three times daily for 7 days in the treatment of bacterial conjunctivitis. Drugs R D. 2013;13:243-52.

8. Bennett S, Carman WF, Gunson RN. The development of a multiplex real-time PCR for the detection of herpes simplex virus 1 and 2, varicella zoster virus, adenovirus and Chlamydia trachomatis from eye swabs. J Virol Methods. 2013;189:143-7.

9. Moore C, Gatica L, Jones T, et al. Collect, boil and amplify-a simple approach for the detection of three common viruses associated with epidemic keratoconjunctivitis, conjunctivitis and dendritic ulcers. J Virol Methods. 2013;189:238-41.

10. Chaurasia S, Ramappa M, Murthy SI, et al. Chronic conjunctivitis due to Mycobacterium tuberculosis. Int Ophthalmol. 2013;34:655-60.

11. Boto-de-Los-Bueis A, Romero Gómez MP, Del Hierro Zarzuelo A, et al. Recurrent ocular surface inflammation associated with human herpesvirus 6 infection. Eye Contact Lens. 2013 [Epub ahead of print].

12. Chen W, Plewig G. Human demodicosis: revisit and a proposed classification. Br J Dermatol. 2014;170:1219-25.

13. Kosik-Bogacka DI, Lanocha N, Lanocha A, et al. Demodex folliculorum and Demodex brevis in healthy and immunocompromised patients. Ophthalmic Epidemiol. 2013;20:159-63.

14. Galea M, Sharma R, Srinivasan S, et al. Demodex blepharitis mimicking eyelid sebaceous gland carcinoma. Clin Experiment Ophthalmol. 2014;42:208-10.

15. Yun SH, Levin F, Servat J. Demodex folliculitis presenting as periocular vesiculopustular rash. Orbit. 2013;32:370-1.

16. • Hom MM, Mastrota KM, Schachter SE. Demodex. Optom Vis Sci. 2013;90:e198-205. This paper demonstrates the high prevalence of demodex.

17. Patel KG, Raju VK. Ocular demodicosis. W V Med J. 2013;109:16-8.

18. Anane S, Malek I, Kamoun G, et al. Phthiriasis palpebrarum: diagnosis and treatment. J Fr Ophthalmol. 2013;36:815-9.

19. Mastrota KM. Method to identify Demodex in the eyelash follicle without epilation. Optom Vis Sci. 2013;90:e172-4.

20. Koo H, Kim TH, Kim KW, et al. Ocular surface discomfort and Demodex: effect of tea tree oil eyelid scrub in Demodex blepharitis. J Korean Med Sci. 2012;27:1545-9.

21. Salem DA, El-Shazly A, Nabih N, et al. Evaluation of the efficacy of oral ivermectin in comparison with ivermectin-metronidazole combined therapy in the treatment of ocular and skin lesions of Demodex folliculorum. Int $\mathbf{J}$ Infect Dis. 2013;17:e343-7.

22. Tavakoli M, Bagheri A, Faraz M, et al. Orbital cellulitis as a complication of mandibular odontogenic infection. Ophthal Plast Reconstr Surg. 2013;29:e5-7.

23. Elmann S, Pointdujour R, Blaydon S, et al. Periocular abscesses following brow epilation. Ophthal Plast Reconstr Surg. 2012;28:434-7.

24. Merino-Inglesias A, Montero JA, Calabuig-Goena M, et al. Orbital apex syndrome secondary to herpes zoster virus infection. 2014.

25. Wong AC, Mak ST. Preseptal cellulitis in a child caused by Megacopta centrosignatum. J AAPOS. 2012;16:577-8.

26. Papakostas TD, Lee NG, Lefebvre DR, et al. Endogenous panophthalmitis with orbital cellulitis secondary to Escherichia coli. Clin Experiment Ophthalmol. 2013;41:716-8.

27. Ramey NA, Burkat CN. Arcanobacterium hemolyticum orbital cellulitis: a rare but aggressive disease. Ophthal Plast Reconstr Surg. 2013;29:e69-72.
28. Naeem M, Rahimnajjad NA, Majeed I, et al. Double trouble: preseptal cellulitis due to two species with multidrug resistance. Head Face Med. 2013;9:17.

29. Lei TH, Huang YC, Chu YC, et al. Orbital cellulitis caused by community-associated methicillin-resistant Staphylococcus aureus in a previously healthy neonate. J Microbiol Immunol Infect. 2013;46:136-8.

30. Moon HR, Park GH, Jung JM, et al. A case of anaplastic largecell lymphoma mimicking preseptal cellulitis. Clin Exp Dermatol. 2014;39:77-8.

31. Pine RR, Clark JD, Sokol JA. CD56 negative extranodal NK/Tcell lymphoma of the orbit mimicking orbital cellulitis. Orbit. 2013;32:45-8.

32. Nair AG, Kaliki S, Ali MJ, et al. Intraocular malignant melanoma of the choroid presenting as orbital cellulitis. Int Ophthalmol. 2014;34:647-50.

33. Bagheri A, Abrishami A, Karimi S. Acute myelogenous leukemia mimicking fulminant periorbital cellulitis. J Ophthalmol Vis Res. 2013;8:380-2.

34. Alkatan HM, Chaudhry I, Alayoubi A. Mature teratoma presenting as orbital cellulitis in a 5-month-old baby. Ann Saudi Med. 2013;33:623-6.

35. Upendran MR, McLoone E. Delayed resolution of eyelid swelling in preseptal cellulitis in a child: beware of causing occlusion amblyopia. BMJ Case Rep. 2013 [Epub ahead of print].

36. Pushker N, Tejwani LK, Bajaj MS, et al. Role of oral corticosteroids in orbital cellulitis. Am J Ophthalmol. 2013;156:178-83.

37. Mathew AV, Craig E, Al-Mahmoud R, et al. Paediatric postseptal and pre-septal cellulitis: 10 years' experience at a tertiarylevel children's hospital. Br J Radiol. 2014;87:20130503. doi:10. 1259/bjr.20130503.

38. Chandravanshi SL, Mishra V. Acute suppurative bacterial dacryoadenitis: a rare case report. Eur J Ophthalmol. 2014 [Epub ahead of print].

39. - Toledano N, Tit-Liviu Stoica B, Genol Saavedra I, et al. Tuberculous dacryoadenitis unveils HIV infection. Can J Ophthalmol. 2013;48:128-30. This paper highlights atypical ocular adnexal infections as a reason to consider evaluation for immune deficiency.

40. Almaloitis D, Nakos E, Siempis T, et al. A para-canalicular abscess resembling an inflamed chalazion. Case Rep Ophthalmol Med. 2013;2013:618367. doi:10.1155/2013/618367.

41. Manderwad GP, Kodiganti M, Ali MJ. Cardiobacterium hominisinduced acute dacryocystitis and lacrimal abscess. Indian $\mathrm{J}$ Ophthalmol. 2014;62:495-7.

42. - Almeida RA, Narikawa S, Tagliarini JV, et al. Dacryostenosis due to Paracoccidioides brasiliensis in a patient with an unnoted HIV-1 infection. Can J Ophthalmol. 2013;48:e61-2. This paper highlights atypical ocular adnexal infections as a reason to consider evaluation for immune deficiency.

43. Celik T, Kosker M, Turkoglu EB. Unilateral acute dacryocystitis associated with oculoglandular tularemia: a case report. Semin Ophthalmol. 2013;28:91-3.

44. Carruth BP, Wladis EJ. Orbital abscess from dacryocystitis caused by Morganella morganii. Orbit. 2013;32:39-41.

45. Ali MJ, Joshi SD, Naik MN, et al. Clinical profile and management outcome of acute dacryocystitis: two decades of experience in a tertiary care eye care center. Semin Ophthalmol. 2013 [Epubahead of print].

46. Sun Y, Wang H, Wang Y, et al. Endonasal endoscopic treatment of recurrent dacryocystitis. Cell Biochem Biophys. 2013;67:1441-4.

47. Eshraghi B, Hashemian H, Fard MA, et al. Lacrimal sac empyema incision and drainage followed by early external dacryocystorhinostomy. Orbit. 2013;32:278-80. 
48. Ari S, Cingu K, Sahin A, et al. The outcomes of surgical treatment in fistulous dacryocystitis. Eur Rev Med Pharmacol Sci. 2013; 17:243-6.

49. - Costin BR, Costin CE, Wall PB, et al. Severe neutropenia presenting with Candida albicans eyelid abscess in a one-year old child. Ophthal Plast Reconstr Surg. 2014 [Epub ahead of print]. This paper highlights atypical ocular adnexal infections as a reason to consider evaluation for immune deficiency.
50. • Chao J, Yumei Z, Zhigun W, et al. Multidrug-resistant bacteria induce recurrent keratoconjunctivitis in a patient with common variable immunodeficiency: case report and literature review. Cornea. 2013;32(Suppl 1):S39-42. This paper highlights atypical ocular adnexal infections as a reason to consider evaluation for immune deficiency. 